\title{
FORMAÇÃO DE RESIDENTES MULTIPROFISSIONAIS EM SAÚDE: LIMITES E CONTRIBUIÇÕES PARA A INTEGRAÇÃO ENSINO-SERVIÇO
}

\section{TRAINING OF MULTIPROFESSIONAL RESIDENTS IN HEALTH: LIMITS AND CONTRIBUTIONS FOR TEACHING- SERVICE INTEGRATION}

\section{FORMACIÓN DE RESIDENTES MULTIPROFESIONALES EN SALUD: LÍMITES Y CONTRIBUCIONES PARA LA INTEGRACIÓN ENSEÑANZA-SERVICIO}

Amanda Lemos Mello ${ }^{1}$, Marlene Gomes Terra ${ }^{2}$, Elisabeta Albertina Nietsche ${ }^{3}$, Daiana Foggiato Siqueira ${ }^{4}$, Janaina Lunardi Canabarro ${ }^{5}$, Cristiane Trivisiol Arnemann 6 .

\section{RESUMO}

Objetivo: analisar os limites e contribuições na formação dos residentes multiprofissionais em saúde para a integração ensino-serviço. Método: pesquisa qualitativa, realizada com 13 docentes vinculados a um Programa de Residência Multiprofissional em Saúde, os quais participaram do estudo por meio de entrevistas semiestruturadas. Os dados foram analisados mediante análise temática de Minayo e os princípios éticos foram respeitados. Resultados: a formação dos residentes é permeada por limitações envolvendo a falta de trabalhadores nos serviços de saúde que contemplem todos os núcleos profissionais propostos pelo Programa e o estranhamento do papel dos residentes ao serem integrantes provisórios das equipes de saúde. Para tanto, a valorização das atividades realizadas a partir da inserção dos residentes tem contribuído para a atenção à saúde e para os serviços de saúde. Conclusão: salienta-se, como contribuição, a discussão da temática de formação profissional, tendo em vista a importância de refletir sobre as transformações das práticas em saúde na perspectiva multiprofissional e de acordo com as necessidades locorregionais.

Descritores: Saúde pública; Prática profissional; Pesquisa qualitativa; Internato não médico; Capacitação Profissional; Serviços de Integração Docente-Assistencial.

\begin{abstract}
Objective: to analyze the limits and contributions in the training of multiprofessional residents in health for teaching-service integration. Method: qualitative research carried out with 13 teachers linked to a Multiprofessional Health Residency Program, who participated in this study through semi-structured interviews. The data were analyzed through Minayo's thematic analysis and the ethical principles were respected. Results: Residents' training is permeated by limitations involving the lack of workers in the health services to cover all the professional centers proposed by the Program and the estrangement of the residents' role when serving as provisional members of the health teams. To that end, the valuation of the activities performed through the residents' insertion has contributed to health care and to the health services. Conclusion: as a contribution, the discussion about vocational training is highlighted, considering the importance of reflecting on the transformations of health practices in the multiprofessional perspective and according to local and regional needs.

Descriptors: Public health; Professional practice; Qualitative research; Internship nonmedical; Professional training; Teaching care integration services.
\end{abstract}

\section{RESUMEN}

Objetivo: analizar los límites y contribuciones en la formación de los residentes multiprofesionales en salud para la integración enseñanza-servicio. Método: investigación cualitativa, realizada con 13 docentes vinculados a un Programa de Residencia Multiprofesional en Salud, los cuales participaron del estudio por medio de entrevistas semiestructuradas. Los datos fueron analizados por medio del análisis temático de Minayo y los principios éticos fueron respetados. Resultados: la formación de los residentes está impregnada por limitaciones que involucran la falta de trabajadores en los servicios de salud que contemplan todos los núcleos profesionales propuestos por el Programa y el extrañamiento del papel de los residentes al seren integrantes provisionales de los equipos de salud. Para ello, la valorización de las actividades realizadas a partir de la inserción de los residentes ha contribuido a la atención a la salud y a los servicios de salud. Conclusión: se destaca como contribución, la discusión de la temática de formación profesional, teniendo en vista la importancia de reflexionar sobre las transformaciones de las prácticas en salud en la perspectiva multiprofesional y de acuerdo con las necesidades locorregionales.

Descriptores: Salud pública; Práctica profesional; Investigación cualitativa; Internado no médico; Capacitación profesional; Servicios de integración docente asistencial.

${ }^{1}$ Graduada em Enfermagem. Doutoranda em Enfermagem pela Universidade Federal de Santa Maria. ${ }^{2}$ Graduada em Enfermagem. Pós-doutora em Enfermagem pela Universidade Federal do Rio Grande do Sul. Docente na Universidade Federal de Santa Maria. ${ }^{3}$ Graduada em Enfermagem. Doutora em Enfermagem pela Universidade Federal de Santa Catarina. Docente na Universidade Federal de Santa Maria. ${ }^{4}$ Graduada em Enfermagem. Doutoranda em Enfermagem pela Universidade Federal de Santa Maria. ${ }^{5}$ Graduada em Enfermagem. Mestre em Enfermagem pela Universidade Federal de Santa Maria. ${ }^{6}$ Graduada em Enfermagem. Pós-Doutoranda em Enfermagem pela Universidade Federal de Santa Maria. Docente na Universidade Federal de Santa Maria.

Como citar este artigo:

Mello AL, Terra MG, Nietsche EA, et al. Formação de Residentes Multiprofissionais em Saúde: Limites e Contribuições para a Integração Ensino-Serviço. 2018; 8: e2567. [Access__]; Available in:___ DOI: 


\section{INTRODUÇÃO}

As práticas de saúde revelam iniciativas permanentes em busca de aproximar a formação profissional de acordo com diferentes necessidades locorregionais e das prioridades de saúde.

Essas iniciativas destacam-se de forma mais evidente nos últimos dez anos, a partir da articulação entre ministérios da Educação (MEC) e da Saúde (MS) e concretizam-se com a proposição das Diretrizes Curriculares Nacionais (DCN) na área da saúde, implementação de políticas, programas e projetos na busca de aproximar as instituições de ensino e serviços de saúde ${ }^{(1)}$.

Diante deste contexto, os Programas de Residência Multiprofissional em Saúde (PRMS) emergem como estratégia para a educação no mundo do trabalho e para o trabalho. Apoiados no processo de ensino, aprendizado e trabalho in loco nas instituições de saúde, possuem como atributo, a formação de profissionais segundo as necessidades locais, isto é, com habilidades e especialidades específicas conforme a deficiência regional| $^{(2)}$.

Esses programas têm como objetivo a formação profissional voltada para uma atuação diferenciada no Sistema Único de Saúde (SUS), com a perspectiva de que os trabalhadores são seres instituintes no processo de trabalho em saúde, impulsionando a construção colaborativa, o trabalho em equipe, a Educação Permanente em Saúde (EPS) e a reorientação das lógicas tecnoassistenciais promovidas pelas mudanças das práticas pedagógicas ${ }^{(3)}$. Com vistas a fortalecer o movimento de mudanças na formação em saúde no Brasil, os PRMS têm sido um exemplo de articulação entre a formação e o trabalho ${ }^{(4)}$.

Entende-se que, a integração ensinoserviço pode ser compreendida como um trabalho coletivo, pactuado, articulado e integrado entre as pessoas que estão envolvidas nos cursos de formação na área da saúde com trabalhadores que compõem as equipes dos serviços dessa esfera ${ }^{(5)}$. Assim, justifica-se este estudo pois, ao compreender que as relações que se estabelecem entre as pessoas poderão contribuir com uma assistência à saúde de qualidade e, por conseguinte, na consolidação dos princípios do SUS, faz-se necessário reconhecer os aspectos que interferem na formação dos residentes multiprofissionais e os serviços de saúde ${ }^{(6)}$.
Além disso, a transformação das práticas de ensino, cuidado e gestão na área da saúde implicam que a integração ensino-serviço ocorra no sentido de facilitar o processo de formação profissional na área da saúde, em consonância com as diretrizes e princípios do SUS ${ }^{(7-8)}$. Para isso, questiona-se: quais os limites e possibilidades na formação dos residentes multiprofissionais em saúde para a integração ensino-serviço? É nesse contexto que se propõe este estudo, cujo objetivo é analisar os limites e contribuições na formação dos residentes multiprofissionais em saúde para a integração ensino-serviço.

\section{MÉTODO}

Trata-se de um estudo exploratório e descritivo, de abordagem qualitativa, realizado em um Programa de Residência Multiprofissional e Integrada em Saúde (PRMIS), na região Sul do Brasil. O PRMIS está vinculado a uma instituição federal de ensino superior e possui as ênfases e as suas áreas de concentração: Atenção Básica/Estratégia de Saúde da Família; CrônicoDegenerativo; Materno-Infantil; Onco-Hematologia; Saúde Mental e Vigilância em Saúde. E contempla os núcleos profissionais: Educação Física, Enfermagem, Farmácia, Fisioterapia, Fonoaudiologia, Nutrição, Odontologia, Serviço Social e Terapia Ocupacional, nos quais estavam vinculados 141 residentes em saúde, no período da pesquisa.

O total de docentes integrantes da RMS eram 25. Desses, apenas 17 respondiam aos critérios de inclusão e exclusão. Dessa forma, buscou-se uma representação mínima de cada núcleo profissional e área de concentração, em que o método de seleção foi sorteio. Assim, participaram do estudo 13 docentes, que atenderam os seguintes critérios de inclusão: ser docente do quadro efetivo vinculado à instituição de ensino superior e estar atuando como tutor (sua função caracteriza-se por atividade de orientação acadêmica de preceptores e residentes) ou preceptor (sua função caracterizase por supervisão direta das atividades práticas realizadas pelos residentes nos serviços de saúde onde se desenvolve o programa) em uma das seis ênfases/áreas de concentração do PRMS. Vale ressaltar que, como havia, por vezes, escassez de preceptores nos serviços de saúde, os docentes do PRMIS realizavam essas atividades e deveriam estar atuando no mínimo um ano no período da produção de dados. $\mathrm{O}$ critério temporal de atuar 
no PRMIS, há no mínimo um ano, foi estabelecido por entender que, nesse período, o docente melhor compreende a realidade e a formação dos residentes, encontrando-se ambientado com as práticas educativas. E, como exclusão, foram considerados aqueles que estivessem em licença de qualquer natureza ou em férias no período da produção dos dados.

A escolha dos docentes ocorreu de modo aleatório, por sorteio. Justifica-se a seleção dos docentes, visto que estes, no programa estudado, configuram-se como um mediador nas relações postas nessa modalidade de ensino, além de serem responsáveis pelo eixo teórico e teóricoprático do PRMS.

A produção dos dados foi realizada nos meses de março a julho de 2015 por meio de entrevista semiestruturada. Essa foi composta por uma questão disparadora: "conte-me como você percebe a integração ensino-serviço na formação dos residentes em saúde". Demais questões foram lançadas conforme o andamento das entrevistas, como: "quais as dificuldades e facilidades que você encontra ao trabalhar com integração ensino serviço na RMIS?".

Para a operacionalização das entrevistas, a pesquisadora convidou os docentes anteriormente via e-mail e/ou contato telefônico. A partir da confirmação dos participantes, foram pactuados horários e locais mais convenientes. As entrevistas apresentaram duração média de 40 minutos, foram áudio-gravadas mediante a autorização dos participantes da pesquisa e transcritas na íntegra pela pesquisadora principal. Todos os participantes foram esclarecidos dos objetivos do estudo e assinaram o Termo de Consentimento Livre e Esclarecido.

A produção dos dados foi encerrada quando houve saturação desses, determinada pela repetição de respostas e alcance satisfatório dos objetivos propostos ${ }^{(9)}$.

Os dados foram submetidos à análise de conteúdo temática da proposta operativa de Minayo, caracterizada por dois momentos operacionais. O primeiro corresponde à fase exploratória da investigação. E o segundo, denominado de interpretativo, foi realizado a partir do encontro com as informações, que se dividiu na ordenação e classificação dos dados e a análise propriamente dita, a qual foi fundamentada em uma perspectiva multirreferencial $^{(10)}$.

A pesquisa foi Aprovada pelo Comitê de Ética em Pesquisa com Seres Humanos, de uma instituição de ensino superior, sob o Parecer № 932528 e CAAE no 40246414.4.0000.5346, sendo respeitadas as normas da legislação brasileira para pesquisas com seres humanos, Resolução no 466/2012. A fim de preservar o anonimato dos participantes, as falas foram identificadas com a letra $D$ (Docente), seguidos de números arábicos (D1, D2, D3 e, assim, consecutivamente).

\section{RESULTADOS E DISCUSSÃO}

A partir da análise qualitativa dos dados, emergiram duas categorias a partir dos seus temas e falas: Categoria 1 . Limites da integração ensino-serviço na formação dos Residentes Multiprofissionais em Saúde, a partir dos temas "Falta de trabalhadores nos serviços de saúde que contemplem todos os núcleos profissionais" e "Provisoriedade do residente". E Categoria 2, Contribuições da Integração ensino-serviço para a formação dos Residentes Multiprofissionais em Saúde, com os temas "contribuições nos serviços de saúde" e "contribuições na atenção à saúde".

Ao que se refere à primeira categoria temática, limites da Integração ensino-serviço na formação dos Residentes Multiprofissionais em Saúde, evidenciou-se que esta formação é permeada por limitações envolvendo a falta de trabalhadores nos serviços de saúde que contemplem todos os núcleos profissionais propostos pelo PRMS e o estranhamento do papel dos residentes ao serem integrantes provisórios das equipes de saúde.

Figura 1 - Primeira Categoria temática, temas e falas de acordo com a análise temática.

\begin{tabular}{|l|l|}
\hline \multicolumn{1}{|c|}{ Categorias temáticas } & \multicolumn{1}{c|}{ Temas e falas } \\
\hline \multirow{4}{*}{$\begin{array}{l}\text { Limites da Integração ensino- } \begin{array}{c}\text { Falta de trabalhadores nos serviços de saúde } \\
\text { que contemplem todos os núcleos profissionais }\end{array} \\
\text { serviço na formação dos } \\
\begin{array}{l}\text { Residentes Multiprofissionais em } \\
\text { Saúde }\end{array}\end{array}$} & $\begin{array}{l}\text { "Eles são técnicos. A atuação deles é como se fosse de um técnico ainda que exista uma } \\
\text { vontade muito grande que eles não sejam simplesmente técnicos e que deveria haver essa } \\
\text { formação. Mas, sem acompanhamento pedagógico do núcleo, por vezes, isso fica difícil". } \\
\text { (D3) }\end{array}$ \\
\cline { 2 - 2 } & $\begin{array}{l}\text { "Colocar profissões novas em locais que nunca tinham atuado, sem aquele profissional ali } \\
\text { dentro do serviço de saúde foi difícil [...] e, portanto, tem o estranhamento, tem uma }\end{array}$ \\
\hline
\end{tabular}




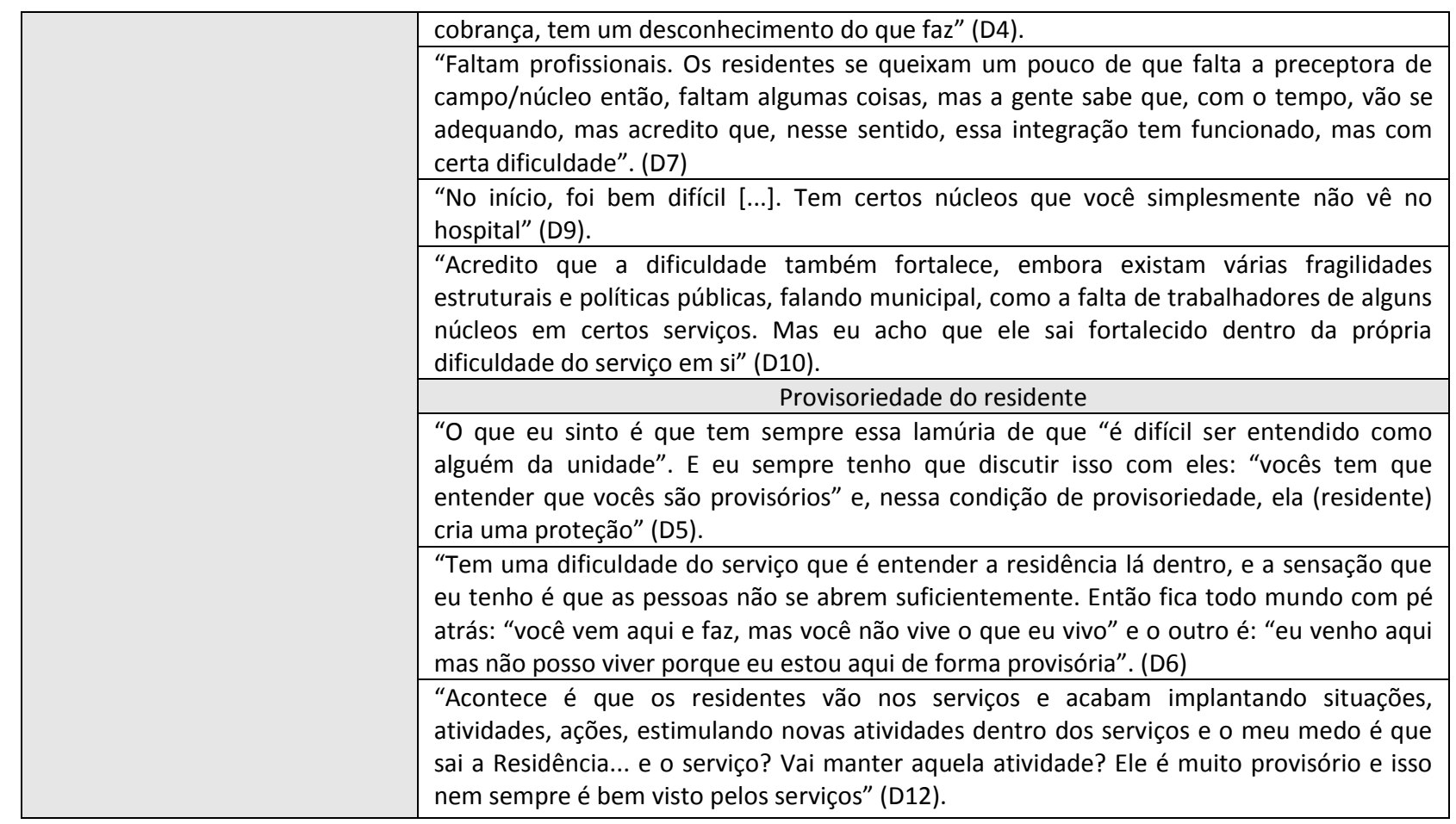

Fonte: Elaborado pelas pesquisadoras, 2017.

A partir dos resultados, evidenciou-se que os residentes perpassam por algumas limitações durante seu processo de formação no PRMS, como a falta de trabalhadores nos serviços de saúde referente ao seu núcleo de formação. Os PRMS possuem como característica central a integração entre as diferentes profissões presentes nos cenários do SUS, buscando desenvolver e potencializar o trabalho e o cuidado integral em equipe, com a devida atenção ao que é comum a todas as áreas e ao que é núcleo profissional específico, tanto de saberes quanto de práticas. Para isso, o residente necessita desenvolver conhecimentos, atitudes e habilidades para atuar de forma interdisciplinar, ou seja, no campo do pensar-fazer comum a todas as áreas profissionais ${ }^{(11)}$.

As ações dos residentes precisam ser sustentadas pela base epistemológica de cada profissão, ou seja, o seu núcleo profissional específico. Para isso, o acompanhamento pedagógico voltado ao núcleo faz-se preciso a fim de auxiliar, na prática, o compromisso com certo saber e com o conjunto de responsabilidades, funções e práticas de cada profissional a fim de indicar uma identidade profissional. Dessa forma, ao agregar o conhecimento do saber específico ao que é comum a todas as profissões, tem-se a ampliação situacional do núcleo, orientada pela necessidade de se cumprirem objetivos acordados em um dado corpo de saber-fazer interdisciplinar ${ }^{(12)}$.
Ao aplicar esses conceitos, tem-se a possibilidade de que os PRMS auxiliem na formação de profissionais dotados de competências para migrar da visão uniprofissional a um estágio mais ampliado, ou seja, a construção da saúde balizada na perspectiva da integração entre pessoas. Assim, ao atuar em equipes multiprofissionais, tem-se a valorização do papel de cada profissional favorecendo, dessa forma, o trabalho coletivo e a oportunidade de novos conhecimentos acerca de outras áreas $^{(4,13)}$.

A formação de profissionais que atuam em equipe é uma maneira de potencializar a atuação colaborativa, visto que a construção destas atuações necessita conter um caráter transformação e progressão das práticas assistenciais frente ao cuidado prestado ao usuário dos serviços de saúde. Neste sentido, o desenvolvimento das atividades permite aos residentes perceberem que a interdisciplinaridade pode colaborar para o progresso da satisfação do usuário que emprega os serviços de saúde públicos, além de aprimorar sua forma de observar e de conhecer as práticas assistenciais ${ }^{(14)}$.

A limitação evidenciada nesta análise de dados pode vir a levantar questionamentos frente ao modo que é valorizada a formação dos residentes em saúde à nível nacional, a escassez de recursos humanos nos serviços de saúde do SUS e, também, o fato de o residente, por vezes, 
ser visto como mão de obra nas equipes de saúde, aspectos esses que podem prejudicar o cumprimento de atividades de campo com os demais residentes ${ }^{(15)}$. Em contrapartida, a inserção dos residentes multiprofissionais em campo de exercício permite a articulação teórico-prática, a capacidade de reflexão e contextualização frente aos obstáculos das experiências de cuidado, que favorecem a obtenção de resultados positivos para a formação. Além disso, o papel do residente enquanto integrantes provisórios das equipes de saúde nas ações assistenciais possibilitam que os mesmos reconheçam diferentes cenários de práticas, além de beneficiar a oxigenação e o amadurecimento do exercício de gestão e cuidado a serem desenvolvidos ${ }^{(16)}$, uma vez que esses benefícios podem ser desencadeados por meio de ações colaborativas, como práticas de EPS ${ }^{(11)}$.

Os docentes enfatizam que essa relação de provisoriedade é um aspecto que pode vir a influenciar no desenvolvimento da integração ensino-serviço. De certa forma, essa situação dificulta a relação entre o trabalhador de saúde, o residente e o contato com o docente. $O$ residente não "deixa correr na veia" aquilo que ele está vivendo na experiência cotidiana. Além do mais, ao serem inseridos nos serviços, no início da sua atuação, eles chegam com força de vontade e desejo de mudança, o que ocasiona o trabalho solitário, limitando as atividades coletivas.
A provisoriedade do residente tem influência direta no desenvolvimento da integração ensino-serviço, visto que a operação principal dessa relação está em traçar um eixo central na formação, com vistas a convergir as atuações de cada um e fortalecer o trabalho cooperado. Para isso, evidencia-se a necessidade de esclarecimento tanto às equipes de saúde quanto aos apoiadores pedagógicos, frente aos objetivos da formação em um PRMS e a importância da sua atuação para a qualificação desses profissionais em formação. Aperfeiçoar os modos de inserção do residente nos cenários de prática profissional poderá contribuir, não somente para a otimização do processo de EPS, mas, principalmente, para a melhoria do cuidado multiprofissional prestado nas instituições ${ }^{(17)}$.

Para tanto, a valorização das atividades realizadas a partir da inserção dos residentes tem contribuído para a atenção à saúde e para os serviços de saúde, como possibilidades conjuntas entre trabalhadores dos serviços, usuários e apoiadores pedagógicos, ou seja, preceptores, tutores e docentes.

Nesse sentido, evidenciou-se a categoria temática: contribuições da atuação do residente nos serviços e na atenção à saúde, cujos temas e falas que geraram estas categorias estão descritas na Figura 2.

Figura 2 - Segunda Categoria temática, temas e falas de acordo com a análise temática.

\begin{tabular}{|c|c|}
\hline Categorias temáticas & Temas e falas \\
\hline \multirow{9}{*}{$\begin{array}{l}\text { Contribuições da } \\
\text { Integração ensino-serviço } \\
\text { para a formação dos } \\
\text { Residentes } \\
\text { Multiprofissionais em } \\
\text { Saúde }\end{array}$} & Contribuições nos serviços de saúde \\
\hline & $\begin{array}{l}\text { "Para além do livro de suporte, isso é produção de vida, de subjetividades, de complexidades, } \\
\text { essa qualidade a gente está construindo. A rede não está posta [...] ela tem falhas [...] mas o } \\
\text { pessoal da residência está ajudando a gente. Estamos parando para pensar nessas falhas" (D1). }\end{array}$ \\
\hline & $\begin{array}{l}\text { "A gente ajuda a colocar aquilo de alguma forma que sirva melhor o serviço, porque os } \\
\text { trabalhadores, eles não conseguem, às vezes, organizar essa parte dos dados, para fazer uma } \\
\text { publicação, não têm esse tempo. Então, por exemplo, indicadores de saúde, indicadores para } \\
\text { avaliar algumas ações em determinados setores, a residência procura observar como vão ser } \\
\text { avaliadas aquelas ações através da utilização de alguns indicadores, porque isso é importante". } \\
\text { (D2) }\end{array}$ \\
\hline & $\begin{array}{l}\text { "O serviço, no geral, qualificou muito com a inserção dos residentes, tanto é que faz seis anos } \\
\text { que trabalho com a residência e a gente é reconhecido" (D8). }\end{array}$ \\
\hline & $\begin{array}{l}\text { "Estamos sendo reconhecidos enquanto residência, existe a nossa profissão dentro do hospital } \\
\text { agora, estamos sendo reconhecidos" (D11). }\end{array}$ \\
\hline & $\begin{array}{l}\text { "A residência veio a contribuir nos serviços aqui do município, porque ela acaba mexendo com a } \\
\text { zona de conforto" (D12). }\end{array}$ \\
\hline & Contribuições na atenção à saúde \\
\hline & $\begin{array}{l}\text { Não se produz saúde de outra forma, não se produz transformação, não se constrói rede. [...] Eu } \\
\text { tenho algumas certezas, claro que, com a experiência clínica, eu vou ter algumas certezas, mas } \\
\text { eu tenho que ter outras dúvidas. Quantas a gente puder compartilhar e construir juntos novas } \\
\text { ideias e reinventar novas ideias através da Residência. (D1) }\end{array}$ \\
\hline & $\begin{array}{l}\text { "Tu quebrar esses muros que separam as profissões para trabalhar a equipe de saúde é difícil, } \\
\text { mas as pessoas agora estão entendendo o que é isso. Não tem como trabalhar saúde sem } \\
\text { equipe de saúde. Sem multiprofissional não tem ganho pra ninguém: nem para o usuário, nem } \\
\text { para o profissional, para ninguém." (D2) }\end{array}$ \\
\hline
\end{tabular}


"A residente pegava aqueles caras e ligava: "nós temos um cara aqui em comum, vamos nos reunir, vamos conversar e vamos traçar um plano?" O que tem de diferente hoje e, eu acho que é um avanço da clínica ampliada: trazer a comunidade para dentro e, isso, a residência auxilia muito". (D6)

"Fizemos uma colônia de férias na vila para as crianças de lá. Então, a residente veio com a ideia e nós montamos um planejamento de horários, atividades. Ela conseguiu o espaço lá com uma igreja que tem perto e eu trouxe a ideia para os alunos da graduação, trouxe uma residente aqui e ela convidou eles". (D13)

Fonte: Elaborado pelas pesquisadoras, 2017.

Destaca-se que, neste estudo, emergiram algumas contribuições dos residentes tanto nos serviços de saúde quanto na atenção à saúde, como a troca de saberes, o fortalecimento do cuidado ofertado aos usuários e a possibilidade do trabalho em rede. $O$ residente multiprofissional é provocado a participar no aprimoramento da equipe em suas atividades diárias como os demais trabalhadores de campo, envolvendo a equipe com as atividades educativas $^{(18)}$. Neste sentido, a RMIS incita e desencadeia os processos de aprendizado, estabelecendo a integração ensino-serviço, uma vez que proporciona ações de troca de saberes e de experiências a partir do seu cotidiano de trabalho.

A partir da atuação dos residentes na atenção à saúde, tem-se possibilidade de fortalecer a Integração Ensino-Serviço e, consequentemente, contribuir para os serviços de saúde no alcance de êxito nas ações que intensifique o cuidado prestado ao usuário proporcionando ganho nas condições de saúde da população. A exemplo desses ganhos, pode-se citar o trabalho colaborativo, em prol da assistência ao usuário em Redes de Atenção à Saúde. Considera-se que esta é uma das propostas do PRMS, pois busca estabelecer relação da prática com a teoria, sendo a integração ensino-serviço uma estratégia de transformação na assistência em saúde. Além disso, atribui ações que têm impactado no processo de trabalho das equipes e nos cursos de formação ${ }^{(19)}$.

Diante disso, percebe-se que a RMS parte de um contexto teórico aliado à imersão do contexto prático para a construção do conhecimento de forma integrada e coletiva frente às vivências e experiências do cotidiano, sendo fundamental a análise sobre a elaboração de novas maneiras que ofereçam a qualificação profissional, havendo a oxigenação das práticas em saúde por meio da EPS oferecida pelo município e gestores dos serviços de saúde, a fim de promover melhorias frente a assistência ${ }^{(11)}$.

Segundo os docentes, pode-se perceber que as práticas de saúde se tornaram ampliadas, vislumbrando a realização de atividades de colaborem com o bem-estar da vida dos usuários a partir de uma prática multiprofissional, favorecendo, dessa forma, a atuação colaborativa entre os profissionais da saúde promovendo a integralidade do cuidado que é fundamental para o fortalecimento do SUS ${ }^{(20)}$.

Nesse sentido, será a partir de estratégias como essas que poderão haver maiores avanços na direção de um sistema de saúde fortalecido, com as instâncias de saúde e educação envolvidas e implicadas para a consolidação das políticas públicas de saúde, condizentes com a demanda local e com vistas à melhoria da formação profissional. Além disso, conforme apontam os docentes, a partir da inserção dos profissionais que foram residentes nos serviços, há um ganho imensurável na melhoria das práticas de cuidado e sua implicação com o Programa de RMS.

Esses cenários de produção do cuidado e produção pedagógica ressignificam a educação na saúde e a propõe como uma relação além do ensino e aprendizagem. É nesse espectro de dimensões do processo de integração em que se estreitam as relações entre docentes, estudantes e trabalhadores de saúde. As relações que se estabelecem entre as pessoas poderão contribuir com uma assistência à saúde de qualidade e, por conseguinte, na consolidação dos princípios do SUS. Todos os elementos que envolvem os processos de mudanças na formação não são neutros, mas permeados por lutas entre posicionamentos e interesses. Também envolvem um caminho de preparação dos responsáveis pela implementação dessas mudanças, estando, em primeira instância, os docentes e os profissionais que atuam na assistência à saúde ${ }^{(21-22)}$.

\section{CONSIDERAÇÕES FINAIS}

Os docentes vinculados ao PRMS deste estudo percebem a integração ensino-serviço na formação dos residentes como um processo que há limitações, como a falta de trabalhadores dos diferentes núcleos nos serviços de saúde e a provisoriedade dos residentes. Mas, ao mesmo 
tempo, os residentes contribuem, significativamente, para os serviços de saúde e a atenção aos usuários devido às ações integradoras que desenvolvem nos cenários de atuação.

Reforça-se, neste estudo, que frente às complexas condições que envolvem a integração ensino-serviço no contexto da formação dos residentes multiprofissionais em saúde, é primordial que se invista em estratégias que facilitem essa relação, como espaços ampliados para discussão e pactuação das atividades voltadas à formação dos residentes.

O estudo teve como limitação ser realizado em um PRMS de uma determinada região geográfica, limitando-se a um contexto social específico. Salienta-se como contribuição, a discussão da temática de formação profissional, tendo em vista a importância de refletir sobre as transformações das práticas em saúde na perspectiva multiprofissional e de acordo com as necessidades locorregionais. Sugerem-se novas pesquisas que objetivem fortalecer as atividades na formação em saúde.

\section{REFERÊNCIAS}

1. Dias HS, Lima LD, Teixeira M. A trajetória da política nacional de reorientação da formação profissional em saúde no SUS. Cienc Saúde Coletiva 2013 jun/18(6):1613-24. DOI: 10.1590/S1413-81232013000600013

2. Cheade MFM, Frota OP, Loureiro MDR, Quintanilha ACF. Residência multiprofissional em saúde: A busca pela integralidade. Cogitare enferm. $2013 \mathrm{jul} / \mathrm{set}$; 18(3):592-5. DOI: 10.5380/ce.v18i3.46360

3. Lobato $C P$, Melchior R, Baduy RS. A dimensão política na formação dos profissionais de saúde. Physis 2012;22(4):1273-91. DOI: 10.1590/S010373312012000400002

4. Silva JC, Contim D, Ohl RIB, Chavaglia SRR, Amaral EMS. Percepção dos residentes sobre sua atuação no programa de residência multiprofissional. Acta Paul Enferm. 2015 mar/abr;28(2):132-8. DOI: 10.1590/1982-019420 1500023

5. Pizzinato A, Gustavo A da S, Santos BRL dos, Ojeda BS, Ferreira E, Thiesen FV, et al. A integração ensino-serviço como estratégia na formação profissional para o SUS. Rev Bras Educ Med. 2012 jan/mar;36(1):170-7. DOI: 10.1590/S0100-55022012000300025

6. Brehmer LCF, Ramos FRS. Experiências de integração ensino-serviço no processo de formação profissional em saúde: Revisão integrativa. Rev Eletr Enf. 2014 jan/mar;16(1):22837. DOI: $10.5216 /$ ree.v16i1.20132

7. Kleba MA. Reflexões sobre o processo de reorientação da formação dos profissionais de saúde no Brasil. In: Kleba ME, Prado ML, Reibinitz KS (Orgs). Diálogos sobre o ensino na saúde: vivencias de reorientação na formação profissional em saúde. Chapecó: Argos, 2016.

8. Vendruscolo C, Trindade LL, Krauzer IM, Prado ML. A inserção da universidade no quadrilátero da educação permanente em saúde: relato de experiência. Texto Contexto Enferm. 2016 mar;25(1):23-7. DOI: 10.1590/0104-0707201600 2530013

9. Minayo MCS. Amostragem e saturação em pesquisa qualitativa: Consensos e controvérsias. Rev Pesq Qual. [Internet]. 2017 [acesso em 2017 nov 10]; 5(7):1-12. Disponível em: http://rpq. revista.sepq.org.br/index.php/rpq/article/view/8 2/59

10. Minayo MCS. O desafio do conhecimento: Pesquisa qualitativa em saúde. 14. ed. São Paulo: Hucitec; 2014.

11. Silva CT et al. Educação permanente em saúde: Percepção de profissionais de uma residência multidisciplinar. Rev Enferm UFSM 2014;3(nesp):627-35. DOI: 10.5902/21797692110 67

12. Campos GWS. Sobre a forma neoliberal de produção dos serviços de saúde no Brasil. In: Gastão WSC. Reforma da reforma: Repensando a saúde. São Paulo: Hucitec, 1992. p. 35-86.

13. Domingos CM, Nunes EFPA, Carvalho BG. Potencialidades da residência multiprofissional em saúde da família: O olhar do trabalhador de saúde. Interface 2015 out/dez;19(55):1221-32. DOU: 10.1590/1807-57622014.0653

14. Maia DB, Souza ETG de, Gama RM, Lima JC, Rocha PC de F, Sassaky Y. Atuação interdisciplinar na atenção básica de saúde: $A$ inserção da residência multiprofissional. Sau. \& Transf. Soc. [Internet]. 2013 [acesso em 2017 out 4];4(1):10310. Disponível em: http://incubadora.periodicos. ufsc.br/index.php/saudeetransformacao/article/v iew/1825

15. Fernandes MNS, Beck CLC, Reiller TH, Viero V, Freitas PH, Prestes FC. Sofrimento e prazer no processo de formação de residentes multiprofissionais em saúde. Rev Gaúcha Enferm. 2015 dez;36(4):90-7. DOI: 10.1590/19831447. 2015.04. $\underline{50300}$

16. Oliveira CF, Guareschi NMF. Formação de profissionais para o SUS: Há brechas para novas formas de conhecimento? In: Ananir PF, Cristiane 
MFR, Vera LP (Orgs). Residências em saúde: Fazeres e saberes na formação em saúde. Porto Alegre: Hospital Nossa Senhora da Conceição, 2010. p. 91-114.

17. Drago LC, Salum RL, Andrade SR, Medeiros M, Marinho MM. A Inserção do residente em enfermagem em uma unidade de internação cirúrgica: Práticas e desafios. Cogitare Enferm. 2013;18(1):95-101. DOI: 10.53 80/ce.v18i1.31313

18. Garcia SD, Ignotti BS, Ciciliato CZ, Vannuchi MTO. Internato de enfermagem: O significado para os internos de uma universidade pública. Rev Enferm UERJ 2014 mar/abr;22(2):1-10. DOI: 10.12957/reuerj.2014.13601

19. Cezar PK, Rodrigues PM, Arpini DM. A psicologia na estratégia de saúde da família: Vivências da residência multiprofissional. Psicol Cienc Prof. 2015jan/mar;35(1):1-10. DOI: 10.15 90/1982-3703000012014

20. Casanova IA, Batista NA, Ruiz-Moreno L. Residência multiprofissional em saúde: Percepção dos residentes sobre a Educação Interprofissional nas práticas colaborativas. In: Anais do 40 Congresso Ibero-Americano em Investigação Qualitativa, 2015; Aracaju. Aracaju: CIAIQ; 2015. p. 368-370.

21. Mourão LC, L'abbate S. Implicações docentes nas transformações curriculares da área da saúde: Uma análise sóciohistórica. Online Braz J Nurs. [Internet]. 2011 [acesso em 2017 out 1]; 10(3): 1-10. Disponível em: http://www.obj nursing.uff.br/index.php/nursing/article/viewFile \$3423/1136

22. Brehmer LCF, Ramos FRS. Experiências de integração ensino-serviço no processo de formação profissional em saúde: Revisão integrativa. Rev Eletr Enf. 2014 jan/mar;16(1):22837. DOI: $10.5216 /$ ree.v16i1.20132

Nota: agência de fomento - Bolsa CAPES Demanda Social. Este artigo é oriundo de uma Dissertação.

Recebido em: 17/10/2017

Aprovado em: 22/05/2018

Endereço de correspondência:

Universidade Federal de Santa Maria

Amanda de Lemos Mello

Avenida Roraima, no 1000, Prédio 26a, sala 1445

CEP: 97105-900 - Santa Maria/RS - Brasil

E-mail: amandamello6@yahoo.com 